\title{
Civilisations
}

Revue internationale d'anthropologie et de sciences

humaines

$57 \mid 2008$

Tourisme, mobilités et altérités contemporaines

\section{Développement touristique et reproduction sociale à l'île Maurice}

\section{Emmanuel Gregoire}

\section{OpenEdition}

\section{Journals}

Édition électronique

URL : http://journals.openedition.org/civilisations/1141

DOI : $10.4000 /$ civilisations. 1141

ISSN : 2032-0442

\section{Éditeur}

Institut de sociologie de l'Université Libre de Bruxelles

\section{Édition imprimée}

Date de publication : 29 décembre 2008

Pagination : 91-106

ISBN : 2-87263-022-8

ISSN : 0009-8140

Référence électronique

Emmanuel Gregoire, « Développement touristique et reproduction sociale à lîle Maurice », Civilisations [En ligne], 57 | 2008, mis en ligne le 29 décembre 2011, consulté le 19 avril 2019. URL : http:// journals.openedition.org/civilisations/1141 ; DOI : 10.4000/civilisations.1141 


\title{
Développement touristique et reproduction sociale à l'île Maurice
}

\author{
Emmanuel GRÉGOIRE
}

Résumé : Après avoir rappelé le contexte historique et sociologique mauricien, l'article décrit les grandes phases du développement touristique de l'île Maurice en montrant qu'il a permis aux grandes familles franco-mauriciennes de maintenir leur contrôle sur l'économie insulaire, et de pallier les défaillances de l'industrie sucrière qui fit leur fortune. Désormais, le tourisme est devenu un élément important de la reproduction sociale locale en confortant le maintien des hiérarchies et par là des inégalités qui prévalaient dans l'économie de plantation dont il prend la relève. Toutefois, une meilleure répartition de ses retombées semble aujourd'hui nécessaire pour préserver la paix sociale et la stabilité politique du pays, l'une et l'autre conditionnant son avenir.

Mots-clés : tourisme, reproduction sociale, inégalités, exploitation du littoral, Maurice.

\begin{abstract}
After a presentation of the historical and sociological Mauritian contexts, this article first recalls the main steps of the development of Mauritian tourism. The text then shows how the touristic development of Mauritius has reinforced the economic power of the great francomauritian families. Tourism plays nowadays a major role in the social reproduction of Mauritius and in the perpetuation of social and economical inequalities that were already prevailing in the former plantation economy, when the main economic resource of the island was sugar. Finally, a better repartition of the economic benefits of tourism seems today to be necessary in order to preserve the national political stability, and social peace, which are both absolutely necessary for local society's future.
\end{abstract}

Key words: tourism, social reproduction, inequalities, coastal exploitation, Mauritius. 
$\mathrm{D}$ epuis les années 1960, les îles tropicales sont devenues une destination touristique très prisée car elles offrent à la fois dépaysement, exotisme et détente. L'île Maurice aux plages de sable blanc bordées d'un lagon aux eaux limpides, à la température clémente, avec des équipements hôteliers de qualité et un accueil chaleureux donne ainsi l'image d'une île paradisiaque. En outre, elle bénéficie de sa stabilité politique, de l'absence de maladies tropicales (le paludisme a disparu), d'animaux et d'insectes dangereux ainsi que de son bilinguisme, fruit de son histoire, et d'un faible décalage horaire avec l'Europe (66\% de ses visiteurs en 2007) et l'Afrique du Sud (9\%).

L'essor du tourisme mauricien a été étroitement lié à celui du transport aérien dont les progrès ont permis de réduire considérablement la durée du voyage notamment avec l'Europe : alors qu'il fallait, autrefois, jusqu'à trois jours pour effectuer le trajet en raison des escales nécessaires au ravitaillement en carburant des avions, celui-ci ne dure à présent qu'une dizaine d'heures. Aussi, le Central Statistics Office (CSO) estime que 906000 personnes se sont rendues en vacances à l'île Maurice en 2007 alors qu'elles n'étaient que 100000 trente ans plus tôt. Le tourisme est donc devenu un des piliers de l'économie nationale aux côtés, d'une part, des exportations de sucre, activité " traditionnelle» de l'île depuis le $19^{\text {e }}$ siècle, et, d'autre part, de l'industrie textile, autre composante du « miracle » économique mauricien. Classée parmi les pays les plus pauvres de la planète au départ des Britanniques (1968), l'île est un des rares pays africains à être sorti du sousdéveloppement pour figurer désormais parmi les pays à revenu intermédiaire $(6000 \$$ / an/habitant en 2007). Ce chiffre est une estimation moyenne qui ne doit toutefois pas occulter les importants écarts qui subsistent entre hauts et bas revenus.

Cet article retrace les grandes phases du développement touristique de l'île en soulignant le rôle joué par les grandes familles franco-mauriciennes qui maintiennent leur contrôle sur l'économie du pays. Il montre ainsi que le tourisme est un élément important de la reproduction sociale, du maintien des hiérarchies et par là des inégalités.

\section{Une société forgée par le sucre}

\section{Le sucre}

Introduite à l'époque néerlandaise (1598-1710), la culture de la canne à sucre qui nécessitait une main-d'œuvre abondante a façonné le peuplement de l'île et sa structure sociale. Son évolution politique et économique récente conforta l'héritage colonial.

L'exploitation du sucre connut un premier essor au cours de la période française. À cette époque (1715-1810), les champs étaient mis en valeur par des esclaves que les planteurs firent venir des côtes du Mozambique, de Madagascar et même de Guinée et du Sénégal : en 1789, ces Africains représentaient près 42000 personnes pour une population de 50000 habitants (Durand 1975). En 1810, les Anglais chassèrent les Français de l'île pour mettre fin aux attaques dont étaient victimes leurs navires de commerce, dévalisés par des corsaires basés à Maurice ${ }^{1}$. En offrant au sucre mauricien de nouveaux débouchés

1. Cette conquête n'a pas été suivie d'une arrivée massive d'Anglais ce qui explique que les Anglo-Mauriciens soient très peu nombreux. Le traité signé avec la France permit, au contraire, aux familles françaises de demeurer dans l'île et de conserver leurs plantations, leurs lois, leur langue, leurs coutumes et leur religion. 
au Royaume-Uni et dans sa colonie indienne, les Britanniques transformèrent l'île en une colonie de plantation alors qu'elle était encore principalement à l'époque une escale sur la route des Indes. Les planteurs français constituèrent alors des domaines plus étendus et recrutèrent de nouveaux esclaves africains et malgaches. Ceux-ci représentaient $75 \%$ de la population en 1835 (Durand 1975).

1835 fut surtout marquée par l'abolition de l'esclavage qui obligea les « Blancs » à trouver une nouvelle source de main-d'œuvre car leurs anciens esclaves désertèrent les lieux de leur servitude pour devenir artisans, pêcheurs, éleveurs ou agriculteurs. Comme à la Réunion, ils furent remplacés par des ouvriers agricoles venus d'Inde, les coolies, recrutés avec des contrats de cinq ans. Paysans pauvres voire petits commerçants ou artisans, ces travailleurs «engagés » étaient, suivant les États d'où ils provenaient, de confession hindoue, tamoul ou musulmane ${ }^{2}$. Cette immigration débuta en 1840 pour s'achever en 1909. Beaucoup de ces journaliers se fixèrent à Maurice si bien que «les Indiens étaient devenus deux fois plus nombreux dans l'île que le reste de la population. En 1901, sur une population totale de 371023 individus, on comptait 259086 Indiens ou Indo-Mauriciens» (Toussaint 1972). À cette immigration, s'ajouta celle, moins importante, de Chinois (Hakkas, Cantonais et Fukinois) qui investirent le commerce de détail des villes et des villages tout en assurant une fonction de banque par le biais du crédit. L'île Maurice devint donc une terre multiethnique, chaque groupe ayant une place particulière dans les rapports de production (Gabriel 1983 : 97-112).

Maurice a donc connu différentes phases de peuplement liées d'une part à la recherche permanente de main-d'œuvre agricole (esclaves africains et malgaches, journaliers indiens) et d'autre part au développement économique local (commerçants chinois). Inversement, la société locale a aussi été marquée par les crises que le sucre a traversées. La transformation de l'île en une économie de plantation fit, d'abord, surgir une classe nouvelle, celle des sucriers, qui supplanta graduellement celle des commerçants et des marins. L'avènement du sucre fit dans un premier temps (jusqu'en 1860) disparaître un grand nombre de petits et moyens propriétaires «blancs » pour donner naissance à de grands domaines. La période suivante (1860-1890) fut, au contraire, marquée par le morcellement des plantations ${ }^{3}$ en raison des difficultés d'exploitation sur des superficies très étendues. D'autres Franco-Mauriciens cessèrent leur activité sous l'effet des cyclones, des maladies qui touchaient la canne (borer) et de la malaria qui sévissait surtout dans les zones littorales. Ils morcelèrent leurs terres au profit d'immigrants d'origine indienne, le plus souvent des sirdars (contremaîtres qui organisaient le travail sur les champs), des recruteurs ou des négociants. Ils favorisèrent ainsi l'émergence d'une nouvelle classe sociale, celle des petits planteurs indo-mauriciens qui, à l'orée du $20^{\mathrm{e}}$ siècle, possédait déjà près de $30 \%$ des terres plantées en canne. À cette époque, de plus en plus de propriétaires de grands domaines renoncèrent au sucre, ne le jugeant plus rentable (son cours étant très bas à Londres). Le démantèlement de ces propriétés permit d'accroître le pouvoir économique et social de la population indienne (Allen 1984 : 340-347) dont certaines franges (Indos-Musulmans) contrôlaient le commerce d'importation des grains (riz) et de la toilerie. Enfin, le mouvement de concentration des sucreries amorcé au $19^{\mathrm{e}}$ siècle se

2. Outre leur religion, les immigrés indiens conservèrent leurs différences culturelles et linguistiques.

3. $90 \%$ des terres étaient aux mains de Franco-Mauriciens (5\% de la population) jusqu'en 1870. 
poursuivit ce qui donna naissance à de grands groupes usiniers franco-mauriciens comme Constance, Médine Sugar Estate, Mon Loisir, Mon Trésor/Mon Désert dont l'activité a pu se diversifier, y compris dans le tourisme, grâce aux revenus tirés de la culture et de la transformation de la canne.

\section{Après le sucre : un nouveau contexte politique et économique}

La première et la seconde guerre mondiale favorisèrent la montée en puissance de marchands musulmans ou d'origine chinoise qui détenaient pratiquement le monopole d'approvisionnement en vivres et en produits manufacturés de l'île, ses relations avec l'Europe étant coupées. La période de l'entre-deux-guerres fut ainsi marquée par l'émergence d'une petite élite indo-mauricienne appartenant aux castes supérieures et intermédiaires de la communauté ${ }^{4}$ et qui bénéficiait d'un accès à l'éducation car les Britanniques avaient alors besoin de personnel pour étoffer l'administration. Aux côtés de quelques Créoles ${ }^{5}$, cette élite anima la vie politique en créant des formations comme le parti travailliste (1936) dont la base sociale était constituée de journaliers indiens. Marqué par le clientélisme hindou dès le milieu des années 1950 (Gabriel 1983 : 97-112), ce parti participa aux différentes élections et réclama le départ des Britanniques. Celuici s'effectua le 12 mars 1968, soit sept mois après les élections législatives qui virent la victoire des partisans de l'indépendance. Seul le Parti Mauricien Social Démocrate (PMSD) fondé par Jules Koenig prônait une association avec la Grande-Bretagne. Ce parti anti-indépendantiste rassemblait les minorités créole, chinoise, blanche et musulmane qui craignaient l'hégémonie hindoue une fois l'île indépendante. Une petite bourgeoisie politique indo-mauricienne regroupée au sein du parti travailliste mené par Sir Seewoosagur Ramgolaam prit donc en main les rênes de l'État dans un contexte extrêmement tendu (émeutes raciales et communautaires de janvier 1968).

Depuis lors, Maurice est devenu une démocratie où le pouvoir politique et administratif revient à la communauté indo-mauricienne de confession hindoue (50\% de la population), les Indos-Musulmans (15\%) étant davantage spécialisés dans le négoce. Le pouvoir économique demeure aux mains des Franco-Mauriciens (2\%) qui forment une puissante oligarchie terrienne, industrielle et financière et par là un groupe social privilégié tout comme les Sino-Mauriciens (3\%) très actifs dans le commerce. Enfin, les Créoles $(30 \%)$ dont les origines culturelles ont été en grande partie effacées par la traite restent la couche sociale la plus défavorisée à l'exception d'un groupe d'intellectuels qui a investi la presse. Le sentiment d'avoir été tenu à l'écart du développement du pays entraîne des tensions avec les Indo-Mauriciens accusés d'être responsables de cette exclusion. Ces tensions se transforment parfois en émeutes, comme ce fut le cas, en février 1999, suite à la mort du chanteur créole Joseph Réginald Topize dit Kaya, décédé au commissariat central de Port-Louis après avoir subi des violences policières.

4. Celle-ci est très hiérarchisée étant, comme en Inde, subdivisée en castes.

5. Ce terme désigne les descendants des esclaves africains et malgaches ainsi que la population née de différents métissages, notamment français et africains. Les Créoles sont de fervents catholiques et leur langue est parlée par la quasi-totalité des Mauriciens. 
Ce rapide tableau de la société mauricienne souligne sa diversité culturelle et son hétérogénéité sociale. De là, naissent de multiples tensions intercommunautaires : en bas de l'échelle sociale, la classe moyenne et populaire indo-mauricienne s'oppose aux Créoles tandis qu'en haut, la couche privilégiée de la communauté indo-mauricienne se confronte au monde des affaires franco-mauriciens voire sino-mauriciens.

\section{Le développement du tourisme mauricien}

Le développement du tourisme mauricien s'est opéré en trois grandes phases (19451985, 1985-2000, 2000-2007) qui en ont fait une activité représentant, selon le CSO, près de $10 \%$ de la population active en 2007 (plus de 50000 emplois directs et indirects confondus), $8,5 \%$ du P.I.B. (4,1\% en 1994) et plus de 40 milliards de roupies de recettes (environ un milliard d'euros) soit la seconde source de rentrées de devises du pays après la zone franche industrielle.

\section{Les débuts (1945-1985)}

L'arrivée des premiers touristes date de l'après-guerre où quelques Anglais s'arrêtaient dans l'île avant de poursuivre leur périple dans l'océan Indien. Ils la parcouraient en train, des agents de la Mauritius Governement Railways les attendant dans chaque gare pour leur proposer un gîte. En 1947, il n'y avait que quatre hôtels : le Curepipe Hotel et l'hôtel Golden à Curepipe, l'hôtel National à Port-Louis et le Family Hotel à Vacoas qui accueillait les familles des soldats britanniques affectés à la garnison. En 1952 (1 800 visiteurs d'après les premières statistiques officielles), la société Rogers acheta, via sa nouvelle filiale Mauritius Hotels, le château Mallac, vaste et belle demeure coloniale pour en faire le Park Hotel. Un peu plus tard, le groupe construisit à proximité de l'aéroport de Plaisance un premier hôtel balnéaire (Le Chaland) puis un second sur la côte Ouest, le Morne Plage (1954). Pour promouvoir le tourisme, les Britanniques créèrent le Mauritius Government Tourist Office (1959). L'activité connut alors un premier essor, le nombre de touristes passant de 7700 en 1963 à plus de 36000 en 1971 (Ministry of Tourism, Leisure and External Communications 2006). Cet essor était dû à l'ouverture de nouveaux établissements : après rénovation (1968), le Morne Plage devint l'hôtel le Morne Brabant équipé d'un golf puis Mauritius Hotels ouvrit en 1971 un troisième établissement, cette fois-ci au nord-ouest (Trou aux biches).

Au début des années 1970, une série d'hôtels luxueux virent le jour sous l'impulsion d'Amédée Hugnin Maingard, premier président d'Air Mauritius et dirigeant de Mauritius Hotels. Il fut un des pionniers du tourisme mauricien aux côtés de Sir Gaëtan Duval. dirigeant créole charismatique du PMSD, ce dernier fut vice-Premier ministre, ministre des Affaires étrangères, du Tourisme et de l'Immigration du gouvernement d'Union nationale formé après l'indépendance (1969-1973) ${ }^{6}$. Épicurien, brillant avocat, Sir Gaëtan Duval était lié à la France où il avait de multiples relations dans le monde du spectacle. Pour faire la promotion touristique de son pays, il le fit découvrir à de nombreuses vedettes du cinéma et de la chanson ainsi qu'à des familles princières comme celle de Monaco. L'île Maurice devint une destination en vogue et prestigieuse qui ciblait une

6. Le PMSD accepta de former une coalition pour éviter que le pays ne connaisse une forte instabilité politique et de nouveaux conflits intercommunautaires. 
Île Maurice :zones touristiques

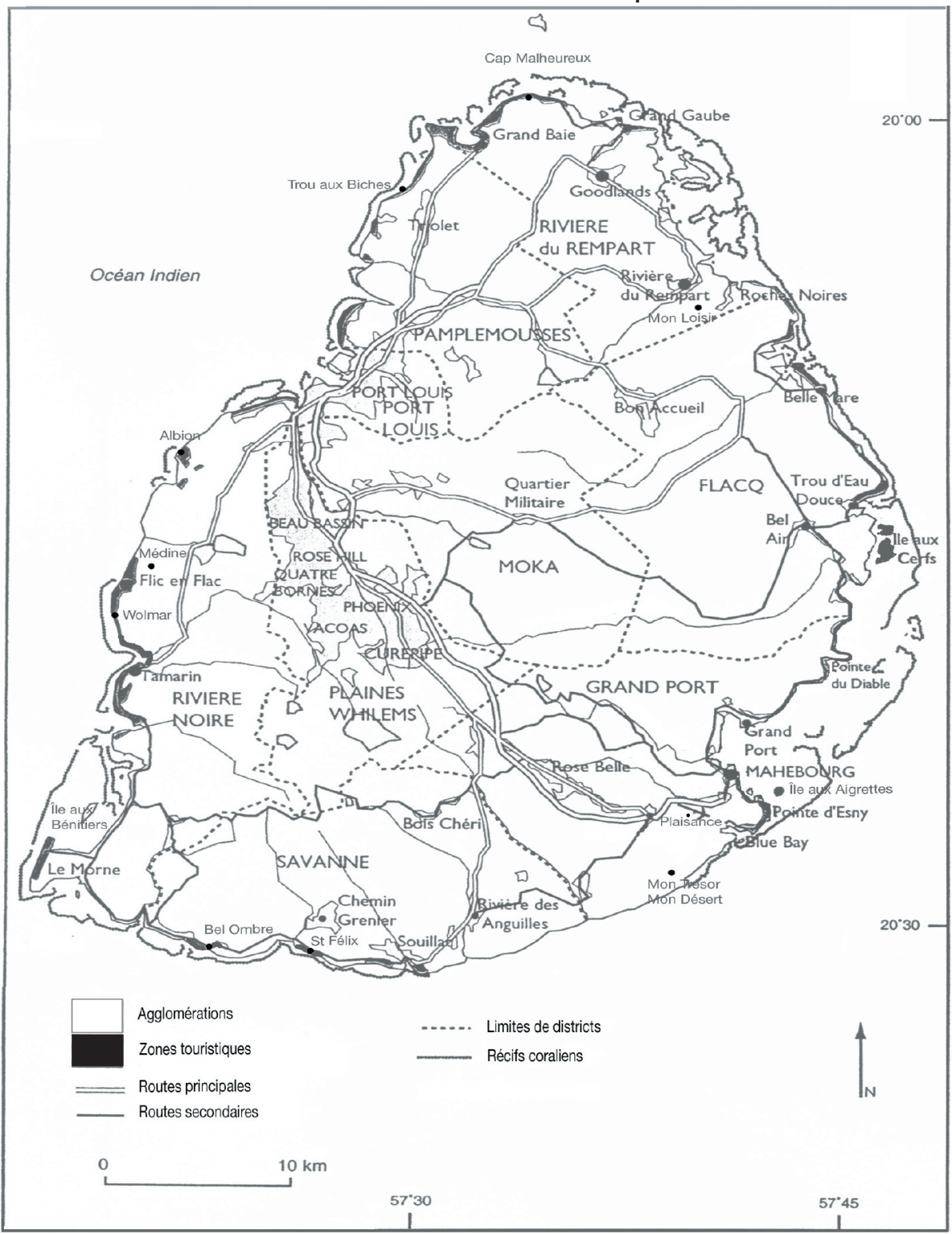

Source : d'après Pébarthe, 2000. 
clientèle haut de gamme et misait sur le fait qu'elle était restée francophone malgré une présence britannique de près de 160 ans. Pour éviter que Mauritius Hotels ne contrôle, seul, l'activité, Sir Gaëtan Duval fit venir le groupe sud-africain Southern Sun de son ami Sol Kerzner. Spécialisé dans l'hôtellerie de luxe, il l'associa au conglomérat Ireland Blyth Limited (IBL) de la famille Dalais pour créer Sun Resorts. Ce groupe installa une série d'hôtels de classe internationale en bordure des plus belles plages comme le Saint-Géran (1975).

Voyant que le tourisme était porteur, des propriétés franco-mauriciennes s'y intéressèrent en profitant du «boom » sucrier de 1974-1975 qui avait pour origine une flambée des cours due une pénurie de sucre sur le marché mondial. Elles investirent une partie de leur surcroît de recettes dans la construction d'infrastructures hôtelières ou la prise de participations dans des groupes hôteliers. Si certaines avaient déjà timidement mis en valeur des terrains proches du littoral sous forme de gîtes, elles se lancèrent cette fois-ci plus franchement dans l'activité en s'inspirant des formules proposées par des groupes étrangers comme le Club Méditerranée. Inauguré en 1973 par son fondateur Gilbert Trigano, ami proche de Sir Gaëtan Duval, le Club fut le premier à proposer la demi-pension et des activités sportives. Fondé en 1882 pour exploiter la canne de la région de Flacq et une sucrerie, le groupe Constance ouvrit ainsi l'hôtel Belle-Mare Plage (1975) qui se substituait à des bungalows. De son côté, Médine Sugar Estate, fondé en 1913, prit des parts dans le complexe hôtelier La Pirogue. Des problèmes de gestion les lui firent revendre en 1983, mais Médine est resté dans le tourisme en ouvrant le parc naturel de Casella (1979) situé au milieu de ses champs de canne. Quant à la propriété Mon Trésor et Mon Désert, elle devint actionnaire de Sun Resorts. Pour mener à bien leurs projets, ces propriétés bénéficièrent de l'appui de la Mauritius Commercial Bank qui est entre les mains de la communauté franco-mauricienne depuis sa fondation en 1838.

Ce souci d'attirer une clientèle privilégiée à forte valeur ajoutée limita le nombre de visiteurs : 75000 en 1975, 148860 en 1985 (Ministry of Tourism, Leisure and External Communications 2006). Il explique que le pays a longtemps été préservé des effets négatifs (dégradation de l'environnement, prostitution, drogue, etc.) de l'arrivée massive de ce que les Mauriciens appellent les «sacs à dos ». Pour l'en empêcher, ils opérèrent une sélection économique et donc sociale de leur clientèle en interdisant la venue de charters et en pratiquant des tarifs élevés au niveau de l'hébergement et du transport aérien.

\section{Un afflux de capitaux et de touristes (1985-2000)}

Le développement du tourisme mauricien s'accéléra à partir du milieu des années 1980 : son chiffre d'affaires passa de 845 millions de roupies (1985) à 14,23 milliards (2000) et le nombre d'hôtels de 55 à 95 (Ministry of Tourism, Leisure and External Communications 2006). Les domaines sucriers s'y intéressèrent davantage car la filière dégageait ses propres profits et bénéficiait des revenus tirés à la fois des exportations de sucre et de l'industrie textile de la zone franche. Si cette dernière démarra lentement, sa croissance fut soutenue à partir de 1983 grâce à l'arrivée de groupes hongkongais, singapouriens et européens et aux investissements des grandes familles franco-mauriciennes. Elles virent là une nouvelle source d'accumulation susceptible de se substituer au sucre, moins lucratif depuis la fin du boom des années 1970.

Les interventions de ces groupes dans le tourisme donnèrent lieu soit à la construction de nouveaux établissements hôteliers, soit au rachat d'infrastructures existantes ou bien 
s'effectuèrent par le biais de l'actionnariat. Une propriété fondée en 1896 et dont les terres se situent dans le sud de l'île (district de Savannah), Union Sugar Estate, investit par exemple dans l'hôtel La Croix du Sud (1987) devenu, depuis lors, le Preskil. En 1998, le groupe Constance y édifia le prestigieux Prince Maurice composé de 89 suites de luxe construites sur la mer grâce à des pilotis en bois et doté d'un parcours de golf (ce groupe possède aussi un hôtel aux Seychelles). Déjà présent dans la manufacture, le commerce, l'assurance, l'imprimerie et d'autres secteurs, le groupe Mon Loisir de la famille Lagesse, fondé en 1939 pour exploiter des plantations du nord-est de l'île, entra quant à lui dans le capital de Naïade Resorts, troisième groupe hôtelier mauricien, puis accrut sa participation en 2003. Nä̈ade Resorts débuta avec le seul hôtel Le Tropical à Trou d'Eau Douce (1990). Depuis lors, il a connu une belle réussite puisqu'il compte à présent six hôtels à Maurice, une luxueuse villa sur l'île des Deux Cocos au large de Blue Bay, un établissement aux Maldives et l'hôtel Desroches aux Seychelles. En peu d'années, Naïade Resorts est devenu le troisième groupe hôtelier mauricien aux côtés de New Mauritius Hotels et de Sun Resorts, deux groupes qui ont dans le même temps multiplié leurs établissements dans l'île. Outre ces trois grands groupes et les domaines sucriers, d'autres opérateurs se tournèrent également vers l'hôtellerie : Food and Allied (FAIL) par exemple, un groupe spécialisé dans l'agro-alimentaire édifia deux hôtels à Port-Louis, puis encore le Telfair à Bel Ombre (2004). Enfin, les groupes étrangers au sein desquels figuraient le groupe réunionnais Apavou et les sociétés de gestion françaises Accor et Méridien s'intéressèrent aussi davantage à Maurice.

En marge de ces grands hôtels, de nombreuses petites structures appelées « campements » (bungalows, appartements, chambres d'hôtes) se développèrent en bord de mer sur des initiatives très diverses. En 2000, une étude (Deloitte and Touche 2002) les évaluait à une capacité de 7000 chambres. Conjugués aux hébergements effectués par les Mauriciens qui accueillent parents et amis résidant à l'étranger, c'était un peu plus de $20 \%$ des touristes qui se logeaient ainsi ce qui représentait plus de $15 \%$ des recettes touristiques du pays ${ }^{7}$. Ces estimations sont imparfaites car ce type d'activité échappe souvent au recensement du ministère du Tourisme, de nombreux bailleurs évitant d'être recensé pour des raisons fiscales. Ce secteur « informel» provoque la grogne des hôteliers qui voient là une concurrence déloyale et des services qui ternissent l'image du pays. À l'inverse, celui-ci dénonce la formule « tout compris » des complexes hôteliers qui les privent de prestations, les touristes trouvant dans les hôtels tout ce dont ils peuvent avoir besoin.

Durant cette période, les agences de voyages européennes étaient chargées de faire venir les touristes à Maurice et bénéficiaient des campagnes de promotion effectuées par la Mauritius Tourism Promotion Authority (MTPA). Ces campagnes eurent d'abord pour cible les clientèles traditionnelles de l'île que sont la France $(26,5 \%$ des touristes selon le CSO) et La Réunion (10,6\%) qui, contrairement à Maurice, ne disposent pas de belles plages de sable hormis à Saint-Gilles. Elles visèrent ensuite de nouveaux marchés comme l'Italie, la Suisse, l'Allemagne puis la Scandinavie, le Japon, l'Australie et l'Asie du SudEst. Le nombre de touristes passa de 115000 (1980) à 291500 (1990) puis à 650000

7. Actualisés, ces chiffres représentent près de 200000 touristes et 5,2 milliards de roupies de recettes (source CSO). 
en 2000, le cap des 500000 visiteurs étant franchi en 1997 (Ministry of Tourism, Leisure and External Communications 2006). Le secteur enregistra alors des taux de croissance élevés (15 à 25\% par an) si bien que s'opéra inévitablement un glissement de la clientèle du haut vers le milieu de gamme, le pays ne pouvant espérer remplir ses hôtels en ne s'adressant qu'à une clientèle fortunée même s'il s'efforce toujours de présenter l'image du luxe.

\section{Toujours plus d'investissements et de touristes (2000-2007)}

Depuis 2000, les investissements se poursuivent et le nombre d'hôtels est en constante augmentation. Le CSO estime ainsi qu'à la fin de l'année 2007 l'île en comptait 97 soit 10857 chambres et 21788 lits. Cinq établissements (non-inclus dans les 97) sont actuellement en rénovation et leurs propriétaires en profitent pour accroître leur capacité d'hébergement. Par comparaison en 1995, les 95 hôtels recensés (Ministry of Tourism, Leisure and External Communications 2006) représentaient 5977 chambres soit une moyenne de 63 chambres par hôtels. Depuis lors, ce chiffre a été multiplié par deux soit 120 chambres en moyenne en 2007 (10 857 chambres pour 97 établissements). Plus que jamais, le tourisme est un des pans essentiels de l'économie du pays car l'industrie sucrière traverse une période difficile : l'Union européenne abolit le protocole sucre qui protégeait les pays ACP (Afrique-Caraïbes-Pacifique), dont Maurice, de la redoutable concurrence de pays comme le Brésil et la Thaïlande (Grégoire et Théry 2007 : 267-282).

Les nouveaux complexes hôteliers et résidentiels se sont implantés principalement dans le sud-ouest de l'île, zone longtemps tenue à l'écart du tourisme en raison de sa vocation agricole. À Bel Ombre, trois hôtels de haute gamme ont été inaugurés en décembre 2004 soit 450 nouvelles chambres et un quatrième à la fin de l'année 2007. À Saint-Félix, la principale plantation sucrière a édifié sur d'anciens champs de canne le Shanti Ananda dont la gestion a été confiée au groupe indien Ananda Resorts. Par ailleurs, New Mauritius Hotels devenu Beachcombers et Sun Resorts ont poursuivi leur essor : le premier possède désormais huit établissements à Maurice et un aux Seychelles tandis que le second en compte cinq et envisage d'acquérir en plus deux établissements aux Maldives. Toute l'île est l'objet de nouveaux projets afin que le pays puisse accueillir deux millions de touristes en 2015 comme l'a souhaité le Premier ministre Navim Ramgoolam. Selon Patrice Hardy, directeur de Naïade Resorts ${ }^{8}$, « il faudra construire six hôtels de 250 chambres par an ». Pour certains observateurs, cet objectif est irréaliste.

Si le cap des 700000 touristes a été franchi en 2002 (Ministry of Tourism, Leisure and External Communications 2006), celui des 800000 n'a pu être dépassé en 2006 en raison du chikungunya. Ce virus, transmis par un moustique dont la piqûre rend très malade quand elle n'est pas mortelle. Le chikungunya a entraîné une forte baisse des arrivées en provenance de France et de La Réunion (respectivement -19,4\% et -9,6\% au cours du premier semestre 2006) où sa prolifération avait été très médiatisée. Cependant, après l'éradication du virus, les flux de touristes reprirent de plus belle : la barre des 900000 arrivées a été franchie en 2007 (906 971) soit une progression de 15,1\% par rapport à 2006 selon le CSO qui estime par ailleurs que les recettes ont crû de 27,3\%

8. Cf. entretien accordé au Mauricien, 16 août 2007. 
passant de 31,94 milliards de roupies en 2006 à 40,68 milliards l'année suivante. Dans ce contexte économiquement favorable, Maurice espère accueillir 975000 visiteurs en 2008. Le pari d'atteindre le million d'arrivées avant 2010 paraît tenable d'autant plus que la multiplication de projets résidentiels «IRS » (Integrated Resorts Scheme) et l'ouverture de l'espace aérien mauricien sont des facteurs qui devraient stimuler le secteur : l'AHRIM (Association des hôteliers et des restaurateurs de l'île Maurice) prévoit de créer 11205 emplois d'ici 2009 grâce à l'ouverture de nouveaux hôtels.

Instaurés en 2002, les IRS permettent l'acquisition de biens immobiliers par des ressortissants étrangers qui obtiennent le statut de résident dès lors qu'ils se portent acquéreurs d'une villa en investissant un minimum de $500000 \$$. Outre des complexes résidentiels, les IRS offrent des infrastructures de grande qualité (piscines individuelles, marina, golfs, restaurants, centres nautiques, de bien-être et de remise en forme, etc.) et en assurent la gestion, l'entretien et le gardiennage. Ces IRS vont donc accueillir une nouvelle clientèle fortunée qui voyagera beaucoup d'où l'importance de la desserte aérienne.

Malgré l'acquisition de nouveaux appareils, l'AHRIM estime qu'Air Mauritius dispose d'une flotte insuffisante pour lui garantir un taux d'occupation de ses établissements satisfaisant y compris en haute saison (octobre à mars). Pour elle, il faut accroître les liaisons aériennes avec l'Europe et donc autoriser la venue de nouvelles compagnies. Cédant à ses pressions, le gouvernement prit l'importante décision d'ouvrir l'espace aérien national (2006). Cette mesure qui risque de fragiliser Air Mauritius désormais confronté à la concurrence a déjà entraîné une forte augmentation des arrivées si bien que nombre d'hôteliers se sont mis à pratiquer la sur réservation (overbooking). Pour répondre à cette nouvelle demande, l'AHRIM envisage d'investir seize milliards de roupies (400 millions d'euros) pour la période 2006-2008 afin de disposer 16500 chambres en 2015.

\section{Tourisme et reproduction sociale}

Le développement touristique de l'île a eu un impact positif en termes de création d'emplois et de distribution de revenus même s'il a fait perdre leur aspect « sauvage " à de nombreux sites en bordure de mer et en a exclu les Mauriciens. Il a également joué un rôle essentiel dans la reproduction de la société mauricienne : comme l'industrie textile auparavant, le tourisme a permis le maintien des hiérarchies économiques, politiques et sociales et par là des inégalités qui prévalaient dans l'économie de plantation dont il prend la relève car ce sont finalement les mêmes personnes qui contrôlent les deux activités à partir de leur capital foncier.

\section{Tourisme et reproduction des milieux d'affaires}

Pendant plus de trente ans, les accords de Lomé ont permis aux grandes propriétés franco-mauriciennes d'écouler leur production sucrière sur le marché européen à un prix nettement supérieur au cours mondial sur lequel elles ne pouvaient s'aligner en raison de coûts de production trop élevés. En se tournant vers le tourisme, ces investisseurs ont cherché à anticiper la fin de cette rente en trouvant de nouvelles sources d'accumulation.

Leurs investissements dans le tourisme bénéficièrent d'une surprotection qui leur permit d'acquérir très vite une position hégémonique. Dans un souci de voir le secteur bénéficier d'abord aux nationaux, l'État leur a en effet accordé des facilités fiscales et douanières et surtout promulgué des décrets (Hôtel Development Certificate de 1972 et Hôtel management Service Certificate de 1982) freinant l'installation des étrangers à 
l'exception de quelques groupes d'envergure internationale comme Le Club Méditerranée. Désormais, les dirigeants du pays n'entravent plus les investissements étrangers car ils estiment qu'ils peuvent contribuer au développement du secteur et créer les emplois indispensables à la lutte contre le chômage et au maintien de la paix sociale (leur part représente à présent près de $30 \%$ du marché).

Les principaux groupes mauriciens présents dans la filière touristique sont directement liés aux grandes propriétés sucrières franco-mauriciennes, notamment les quatre principales familles (Dalais, Espitalier-Noël, Lagesse et de Spéville) qui contrôlent une partie de l'économie du pays. Les autres communautés nationales ne se sont pas, au départ, impliquées dans l'activité ou y ont connu des déboires. À cela, certains observateurs avancent des raisons culturelles : le tourisme et les activités de plage ne font pas partie de la culture des Sino-Mauriciens ni de celle des Indo-Mauriciens. Pour d'autres, leur entrée dans le tourisme a été entravée par leur incapacité à obtenir des financements et par un manque de relations dans les milieux professionnels internationaux mais aussi de savoir-faire. Quelques-uns évoquent enfin un certain complexe à investir l'activité aux côtés des «Blancs»: ce sentiment renvoie à l'histoire de Maurice où les hiérarchies datant de l'esclavage et de l'engagisme continuent d'imprégner la société (Chazan-Gillig 2004 : 64-86).

Néanmoins, la situation a un peu évolué depuis une dizaine d'années. Les SinoMauriciens qui ont déjà depuis longtemps investi la restauration balnéaire et urbaine, possèdent désormais plusieurs établissements hôteliers ou bien sont actionnaires de grands groupes dans lesquels ils ont pris des participations à partir des gains réalisés dans le négoce et l'import-export où ils sont très actifs. Les Indo-Mauriciens ont également perçu tout l'intérêt du secteur (ils interviennent un peu dans la restauration) : Bhunjun and Sons, société spécialisée dans la construction, possède ainsi plusieurs hôtels et a un établissement en construction. D'autres groupes, notamment indo-musulmans, ont aussi pris des participations financières dans des sociétés hôtelières. Toutefois, les SinoMauriciens et les Indo-Mauriciens se sont impliqués dans l'activité uniquement en tant que propriétaires ou actionnaires et non en tant que gestionnaires. Ces tâches sont dévolues à des Franco-Mauriciens ou à des Européens pour des raisons culturelles et de compétences. Enfin, la présence des Créoles dans le secteur touristique reste cantonnée, en ce qui concerne les dirigeants d'entreprise, aux seules agences de voyage (réception des touristes, location de voitures, expéditions marines et terrestres, etc.).

Tout comme l'hôtellerie classique, les projets IRS favorisent les grandes propriétés sucrières car celles-ci détiennent un capital foncier important, notamment des terres proches du littoral. Beaucoup d'entre elles (Médine à Tamarin, CIEL à Beau Champ, etc.) mettent en place ces nouvelles résidences, au nombre d'une vingtaine environ, ce qui représente 4600 villas pour un investissement total de 30 milliards d'euros. Pour " démocratiser » l'économie et faire en sorte que ces IRS ne profitent pas aux seuls FrancoMauriciens, le gouvernement incite les planteurs indo-mauriciens à soumettre des projets comme c'est le cas dans la zone de Flic-en-Flac. Cette initiative semble jusqu'à présent relever davantage d'un vœu politique que d'une réalité économique car les planteurs indo-mauriciens ne disposent ni des capitaux ni du savoir-faire nécessaires pour se lancer dans de telles entreprises.

Malgré un intérêt désormais général, le tourisme reste concentré entre les mains de trois grands groupes: Beachcombers, Sun Resorts qui s'est séparé de son partenaire sud-africain ainsi que Naïade Resorts avec pour actionnaire important le groupe sucrier 
mauricien Mon Loisir. Viennent ensuite Constance Hôtels, FAIL et Veranda Resorts. Ce classement montre que le tourisme demeure une "affaire de Blancs » qui a permis la reproduction de la hiérarchie économique qui prévalait et prévaut encore dans la filière sucrière : les mêmes groupes et individus ou leurs fils contrôlent les deux activités. Plus encore, les concepteurs du tourisme mauricien ont, consciemment ou inconsciemment, reproduit le modèle sociétal local où chaque communauté vit repliée sur elle-même, les relations « interethniques » se limitant pour beaucoup de gens au seul monde du travail. Le tourisme est agencé sur ce modèle d'isolement : les complexes hôteliers forment de véritables ghettos protégés de l'extérieur par des murs et des gardiens. Cette conception est fort éloignée des nouvelles approches comme le tourisme solidaire qui encourage, au contraire, les échanges entre visiteurs et populations locales (Barlet, Collombon et Ribier 2004). À Maurice, les touristes sont déconnectés de la réalité sociale et leurs relations avec les Mauriciens se limitent bien souvent aux seuls employés des hôtels si bien qu'en quittant le pays ils véhiculent l'image d'une société qui vit en parfaite harmonie malgré sa diversité culturelle et religieuse. Ils n'ont évidemment pas vu les quartiers miséreux de Port-Louis ni les taudis de Rivière noire proches des grands hôtels du Morne et n'ont pas conscience des tensions intercommunautaires.

\section{Tourisme et reproduction de l'élite politique et administrative}

Après son indépendance, l'État mauricien a assuré sa construction sur le sucre, seul secteur d'activité qui engendrait des profits et où circulait de l'argent. La petite bourgeoisie politique indo-mauricienne qui se substitua aux Britanniques mit en place tout un système de taxation des exportations de sucre, mais aussi des grandes propriétés. C'est également sur le sucre que ces nouveaux dirigeants assurèrent leur promotion économique et sociale : ministres, hauts fonctionnaires et agents de l'État ponctionnèrent le secteur à des fins d'enrichissement personnel sous forme de prébendes, de pratiques corruptives et de détournements. L'État et le pouvoir politique constituèrent aussi, comme cela s'est souvent produit en Afrique, des sources d'accumulation pour quelques gros commerçants et industriels hindous, musulmans et même créoles qui doivent leur réussite à des mesures politiques, à des marchés octroyés par l'État voire à des combines financières.

À partir de la fin des années 1980, le tourisme devint de plus en plus prospère alors que le sucre était moins lucratif. La classe politique et administrative indo-mauricienne se reporta sur lui pour s'assurer de nouvelles ressources et pallier les défaillances de l'industrie sucrière, imitant en cela la communauté franco-mauricienne. Des hauts responsables du pays vont monnayer leur pouvoir à l'occasion de la délivrance d'autorisations diverses et de la location de terrains en bordure de mer à des fins hôtelières. Les sommes en jeu sont parfois d'un montant très élevé comme une rumeur le laisse entendre à propos des IRS. Sous couvert de leur épouse, de prête-noms - voire parfois en leur nom propre, les responsables politiques réinjectent l'argent ainsi gagné dans le secteur touristique sous forme d'actionnariat dans des groupes hôteliers.

Première entreprise mauricienne par son chiffre d'affaires (près de quatre milliards d'euros en 2006), Air Mauritius dont l'État est avec le conglomérat Rogers le principal actionnaire, a été au cœur du développement touristique de l'île, mais aussi de nombreux scandales financiers. Au départ (juin 1967), la compagnie offrait un service au sol pour les passagers et le fret aérien. En 1972, elle acquit son premier avion affecté à la desserte de Rodrigues, seconde île de la république de Maurice, puis un second en 1975 qui desservait 
La Réunion et enfin son premier long-courrier en 1981. Avec la croissance de sa flotte (actuellement dix Airbus), son réseau de destinations se diversifia vers l'Europe, l'Afrique du Sud, l'Australie et le Moyen-Orient tout en accentuant sa traditionnelle implantation en Inde, à Hong-Kong et Singapour. Air Mauritius achemina donc un nombre croissant de touristes dans l'île et devint une des premières entreprises du pays. Brassant d'énormes sommes d'argent, son histoire a été émaillée de scandales financiers dont la presse s'est fait l'écho et dont la justice s'est saisie. Ces scandales eurent pour origine des détournements de fonds dans lesquels la communauté indo-mauricienne était largement impliquée car elle monopolise la direction et les postes-clés de la compagnie pratiquement depuis sa création. En raison de leur répétition, il serait trop long de relater toutes les affaires qui ont marqué son histoire pour le moins tumultueuse. On peut cependant avancer qu'Air Mauritius fut une source d'enrichissement personnel importante pour plusieurs de ses dirigeants et cadres supérieurs et que ses comptes souffrirent de leurs agissements frauduleux.

La récente décision de libéraliser l'espace aérien national, tout comme l'autorisation donnée aux étrangers de s'installer dans l'île (IRS) traduit l'adoption d'une politique désormais très libérale. Ces deux mesures constituent une rupture politique radicale, les précédents gouvernements s'étant toujours refusés à les prendre au nom d'un certain nationalisme. Pour se justifier, le gouvernement travailliste de Navim Ramgoolam dont le ministre du Tourisme est Xavier-Luc Duval, fils de Sir Gaëtan Duval, argue qu'elle était devenue indispensable. En faisant venir davantage de touristes, le gouvernement espère créer les emplois (le taux de chômage est de 9,2\% en 2007 selon le CSO) qui compenseront les pertes enregistrées dans le sucre et le textile suite à l'abolition du protocole sucre et de l'accord multifibres ( $1^{\text {er }}$ janvier 2005). Néanmoins, certains informateurs interprètent plutôt les décisions prises par le ministre comme une concession faite à l'AHRIM et par là aux milieux d'affaires franco-mauriciens qui souhaitent le minimum d'entraves à leur activité.

\section{Le tourisme face aux problèmes sociaux et environnementaux}

Prenant la relève du sucre, le tourisme constitue donc une nouvelle manne dont profitent les milieux d'affaires franco-mauriciens et la classe politique indo-mauricienne. Les Créoles n'en tirent guère de bénéfices si ce n'est quelques emplois. Si le sucre a longtemps employé une main-d'œuvre nombreuse et peu qualifiée, le tourisme a eu aussi besoin de bras pour assurer le service dans les hôtels et les autres infrastructures. Les « coupeurs de canne » sont peu venus dans le tourisme en raison de leur incapacité à se reconvertir ou parfois en raison de leur âge déjà avancé. Par contre, le tourisme procure aussi des emplois à toute une masse de gens, souvent jeunes, de condition modeste d'origine indo-mauricienne (basses castes) voire métisse (indo-créole, etc.). Les Créoles par contre sont beaucoup moins représentés et lorsqu'ils travaillent dans les hôtels, ils sont le plus souvent affectés aux basses besognes (femme de ménage, manœuvre, etc.). Moins scolarisés et maîtrisant moins bien le français et l'anglais que les autres Mauriciens ${ }^{9}$,

9. Si tous les jeunes Mauriciens sont scolarisés, les Créoles abandonnent assez vite l'école : peu d'entre eux obtiennent le SC (équivalent de la seconde) ou le HSC (niveau du baccalauréat) et rares sont ceux qui intègrent l'université. 
les Créoles fréquentent peu l'école hôtelière qui forme la plupart des employés du secteur touristique. Le tourisme maintient ainsi le système de domination économique et les inégalités sociales établis par l'industrie sucrière, processus imperceptible par les touristes. Toutefois, ce mode d'organisation ne peut fonctionner que si la paix sociale est maintenue.

Si l'activité touristique est vulnérable aux pandémies (chikungunya) et aux catastrophes naturelles (cyclones, tsunamis), elle l'est aussi à la conjoncture nationale (le tourisme kenyan a été sévèrement touché par les troubles intérieurs qui ont affecté le pays en 2007) et internationale (les attentats du 11 septembre 2001 et la guerre en Irak ont entraîné une baisse des flux). À Maurice, le succès de l'industrie touristique repose sur la stabilité politique du pays qui tranche avec la situation de nombreux pays africains régulièrement affectés par des coups d'État. Toutefois, cet équilibre est précaire : des troubles politiques ou sociaux peuvent remettre en cause de longues années d'efforts comme cela aurait pu être le cas lors des émeutes de 1999. Maurice n'est donc pas à l'abri de crises intérieures. Pour les éviter, les tenants de l'activité doivent faire en sorte que le tourisme ne profite pas seulement à une minorité : il y a, semble-t-il, un seuil de tolérance à la poursuite de son développement. Il réside dans le sentiment qu'éprouvent de nombreux Mauriciens tenus à l'écart de ses retombées tout en en supportant les inconvénients et en voyant quelques privilégiés s'enrichir alors qu'ils s'appauvrissent avec le retour de l'inflation et du chômage.

Un autre problème réside dans le déséquilibre croissant entre le nombre total de visiteurs et la population locale. Certains redoutent désormais qu'une partie de la population (celle qui ne profite pas, ou peu des retombées économiques) finisse par manifester un rejet $\mathrm{du}$ tourisme et des touristes. L'AHRIM estime pourtant que l'objectif d'accueillir deux millions de vacanciers en 2015 ne pose aucun problème : répartis sur un an et compte tenu que la durée moyenne d'un séjour est de dix jours, cela ne fera qu'environ 55500 visiteurs en permanence sur l'île soit 4,5\% de sa population (1 250000 habitants répartis sur $1865 \mathrm{~km}^{2}$ ), de plus, concentrés dans des lieux précis ${ }^{10}$. Les incidents ponctuels qui se produisent dans les zones les plus fréquentées contredisent ces arguments : les pêcheurs de la région du Morne se plaignent par exemple de ne pouvoir pêcher en toute quiétude en raison des activités de surf et des bateaux de plaisance. À Saint-Félix, les habitants se sont regroupés en association « $\mathrm{Pa}$ tous nou laplaz » pour protester contre une décision gouvernementale d'accorder à des promoteurs plus de 72000 mètres carrés de plages publiques. Pour répondre à ces réticences, l'État oblige les promoteurs à financer des actions sociales dans leurs zones d'implantation (rénovation et construction d'infrastructures) à hauteur de 25 millions de roupies ${ }^{11}$. Plus encore, il a récemment refusé sous la pression des populations et parfois des écologistes d'avaliser des projets comme ce fut le cas à l'île aux Bénitiers et à l'île aux Deux Cocos.

Si jusqu'à présent les tensions entre autochtones et les projets touristiques demeurent locales (conflits relatifs à des terres convoitées), les propos tenus à propos des IRS («on

10. Les Maldives (350 000 habitants) accueillent près de 600000 touristes par an.

11. Pour ne pas retarder leurs projets, des promoteurs préfèrent indemniser directement les villageois même s'ils estiment que leurs mouvements d'opposition n'ont d'autres buts que d'obtenir des dédommagements injustifiés. 
vend Maurice aux étrangers ») montrent un changement d'échelle : un réel sentiment de rejet s'amorce car l'espace touristique empiète désormais trop sur celui alloué aux Mauriciens. L'amélioration de leurs conditions de vie a fait que le littoral est devenu un espace social qu'ils utilisent durant les week-ends et leurs congés (Magnan 2007). Aussi, leurs ambitions quant à l'usage des « dernières plages disponibles » entrent en conflit avec celles des hôteliers qui entendent les confisquer à des fins touristiques. Alexandre Magnan (2007) a montré que ces problèmes ne se posent pas (encore) d'un point de vue strictement quantitatif mais surtout d'un point de vue qualitatif (attractivité et accessibilité des plages), les complexes hôteliers s'accaparant les derniers beaux sites d'accès facile.

À ces réticences face au développement toujours plus important de l'industrie touristique s'ajoutent des considérations environnementales : l'exploitation intensive du littoral n'entraîne-t-elle pas une saturation de l'espace ? Le linéaire côtier représente 322 kilomètres dont 267 situés à l'arrière d'une barrière récifale trouée de multiples passes, formant ainsi de nombreuses baies et anses (Cazes-Duvat et Paskoff 2004). Les plages de sable blanc convoitées par le tourisme en constituent près des deux tiers (Magnan 2007 : 341-372). Les hôtels et les "campements » occupaient 3,1\% du linéaire disponible en $1975,13 \%$ en 1996 et près de $20 \%$ aujourd'hui. Les emplacements libres se raréfient si bien que les derniers espaces libres sont convoités et les prix flambent. Par ailleurs, des signes de détérioration de l'environnement (dégradation du lagon, érosion des côtes) sont perceptibles et des paysages, auparavant sauvages, ont été défigurés par des complexes hôteliers. Enfin, l'île connaît des problèmes d'approvisionnement en eau en raison de la pression exercée par le secteur touristique (entretien des piscines, des golfs, des gazons, etc.) et les champs de canne.

\section{Conclusion}

Sur l'île Maurice, le tourisme a reproduit les mêmes hiérarchies que celles qui prévalaient dans l'économie de plantation. Il a ainsi conforté l'héritage socio-économique colonial qui continue d'imprégner la société mauricienne encore très marquée par l'esclavage et le travail journalier. Comme le textile auparavant, le tourisme a contribué à maintenir les inégalités et n'a donc pas réduit les barrières sociales, raciales et intercommunautaires. Contrairement à la Thaïlande par exemple, où l'État a favorisé le développement du tourisme domestique (notamment sur des sites historiques majeurs) pour favoriser le sentiment d'unité nationale et pacifier les relations sociales (Evrard 2006 : 151-167), le tourisme à Maurice demeure principalement, en dépit de son ouverture récente à l'écotourisme, essentiellement international et balnéaire. Les visiteurs ne se rendent pas, par exemple, à l'Aapravasi Ghat, lieu où plus de 400000 travailleurs indiens « engagés » ont débarqué, ni au lac sacré de Grand Bassin, ni dans les pagodes de Port-Louis ou sa cathédrale ni enfin dans le musée « l'Aventure du sucre » installé sur l'ancienne usine de Beau Plan.

Le développement touristique de l'île a, certes, créé des emplois et distribué des revenus. Il n'a toutefois pas réduit les inégalités sociales. Les Créoles notamment, plus que les autres communautés nationales, ont été tenus à l'écart de ses retombées comme ils l'ont été en partie du «miracle » économique des années 1980 et 1990. Le sentiment d'exclusion ressenti par une partie de la population, pas seulement d'ailleurs d'origine créole, et la détérioration de ses conditions de vie avec le retour de l'inflation et du chômage pourraient, un jour, mettre en péril la stabilité politique du pays et la paix sociale, atouts 
majeurs du tourisme mauricien et condition sine qua non à son épanouissement. Des troubles intérieurs anéantiraient alors sur-le-champ de longues années d'efforts comme ce fut récemment le cas au Kenya. Maurice ne semble pas à l'abri de ce type de situation compte tenu des multiples tensions qui traversent une société qui, sous de nombreux aspects, paraît « bloquée ».

\section{Références citées}

Allen, Richard, 1984. "Indian Immigrants and the Beginnings of the Grand morcellement (1860-1885)", in Utamah Bissoondoyal (ed.), Indian Overseas: The Mauritain Experience, pp. 340-347. Moka (Mauritius): Mahatma Gandhi Institute.

Barlet, Sandra, Jean-Marie Collombon et Danièle Ribier, 2004. Tourisme solidaire et développement durable, AGRIDOC. Paris : Éditions du GRET.

CAZES-Duvat, Virginie et Roland PASKoff, 2004. Les littoraux des Mascareignes entre nature et aménagement. Paris : L'Harmattan.

Chazan-Gillig, Suzanne, 2004. "The roots of Mauritian multi-culturalism and the birth of a new social contract : Being creole, being autochtone”. Journal of Mauritian Studies. New Series, 2 (1), pp. 64-86.

Deloitte and Touche, 2002. Tourism Development Plan for Mauritius. Port-Louis: Sigma-Ove Arup and Partners, (3 volumes).

DuRAnD, Joyce et Jean-Pierre, 1975. L'île Maurice : quelle indépendance? La reproduction des rapports de production capitalistes dans une formation sociale dominée. Paris : éditions Anthropos.

Evrard, Olivier, 2006. «L'exotique et le domestique. Tourisme national dans les pays du Sud : réflexions depuis la Thaïlande », in Autrepart 40, pp. 151-167. Paris : Armand Colin/IRD.

Gabriel, Claude, 1983. "Communalisme, structures sociales et dépendance économique à l'île Maurice », Politique Africaine, pp. 97-112.

Gregoire, Emmanuel et Hervé Thery, 2007. « L'Ogre et le petit Poucet, le Brésil et l'île Maurice dans le négoce mondial du sucre », L'Espace géographique, 36, pp. 267-282.

Magnan, Alexandre, 2007. « Tourisme et réserves d'espaces pour les pratiques locales sur les littoraux de l'île Maurice », Cahiers d'Outre-Mer, 240 (60), pp. 341-372.

Ministry of Tourism, Leisure and External Communications, 2006. Handbook of Statistical Data on Tourism, 26. Port-Louis: Ministry of Tourism.

PeBARTHe, Hélène, 2000. Littoral mauricien et tourisme, quelles perspectives de développement et de gestion intégrée pour le sud-est de l'île? Paris : GRAFIGEO, collection Mémoires et documents de l'UMR Prodig, 2000-12.

Toussaint, Auguste, 1972. Histoire des îles Mascareignes. Paris : Berger-Levrault. 